\title{
Meningoencephalitis and Listeria monocytogenes, Toxoplasma gondii and Brucella spp. coinfection in a dolphin in Italy
}

\author{
Carla Grattarola ${ }^{1}$, Federica Giorda ${ }^{1}$, Barbara Iulini ${ }^{1}$, Maria Domenica Pintore ${ }^{1}$, \\ Alessandra Pautasso ${ }^{1}$, Simona Zoppi ${ }^{1}$, Maria Goria ${ }^{1}$, Angelo Romano ${ }^{1}$, \\ Simone Peletto ${ }^{1}$, Katia Varello ${ }^{1}$, Fulvio Garibaldi ${ }^{2}$, Giuliano Garofolo ${ }^{3}$, \\ Cristina Esmeralda Di Francesco ${ }^{4}$, Letizia Marsili ${ }^{5}$, Elena Bozzetta ${ }^{1}$, \\ Giovanni Di Guardo ${ }^{4}$, Alessandro Dondo ${ }^{1}$, Walter Mignone ${ }^{6}$, Cristina Casalone ${ }^{1, *}$ \\ ${ }^{1}$ Istituto Zooprofilattico Sperimentale del Piemonte, Liguria e Valle d'Aosta, Via Bologna 148, 10154 Turin, Italy \\ ${ }^{2}$ University of Genova, Dipartimento di Scienze della Terra, dell' Ambiente e della Vita, Corso Europa 26, 16132 Genova, Italy \\ ${ }^{3}$ National and OIE Reference Laboratory for Brucellosis, Istituto Zooprofilattico Sperimentale dell'Abruzzo e del Molise, \\ Campo Boario, 64100 Teramo, Italy \\ ${ }^{4}$ University of Teramo, Faculty of Veterinary Medicine, Località Piano D'Accio, 64100 Teramo, Italy \\ ${ }^{5}$ University of Siena, Dipartimento di Scienze Fisiche, della Terra e dell'Ambiente, Via Mattioli 4, 53100 Siena, Italy \\ ${ }^{6}$ Istituto Zooprofilattico Sperimentale del Piemonte, Liguria e Valle d'Aosta, Via Nizza 4, 18100 Imperia, Italy
}

\begin{abstract}
Listeria monocytogenes, Toxoplasma gondii and Brucella spp. can infect a wide range of species, including humans. In cetaceans, meningoencephalitis has been associated with T. gondii and Brucella spp. infection, whereas to our knowledge, L. monocytogenes infection has not previously been reported. Meningoencephalitis and L. monocytogenes, T. gondii and Brucella spp. were identified by means of both direct and indirect laboratory techniques in an adult female striped dolphin Stenella coeruleoalba found stranded in January 2015 on the Ligurian Sea coast, northwestern Italy. The animal was emaciated, and histopathology disclosed severe meningoencephalitis. The nature of the inflammatory response and intra-lesional protozoa were consistent with a mixed infection by L. monocytogenes, T. gondii and Brucella spp. We believe this is an unprecedented case of infection by 3 zoonotic pathogens and also the first bacteriologically confirmed case report of neurolisteriosis in cetaceans. Cerebral toxoplasmosis and neurobrucellosis may have led to the animal's disorientation and stranding, with L. monocytogenes having likely exacerbated the coinfection leading to the demise of this dolphin.
\end{abstract}

KEY WORDS: Meningoencephalitis · Toxoplasmosis · Listeriosis · Brucellosis · Stenella coeruleoalba

\section{INTRODUCTION}

Listeria monocytogenes is a Gram-positive coccobacillus that causes invasive disease in humans and animals (Haase et al. 2014); it inhabits both populated and pristine environments (Zaytseva et al.
2007), being able to survive for a long time in the sea environment, where it is widespread among invertebrate and vertebrate organisms (Beleneva 2011). Invasive disease has been documented in wild animals, but no listeriosis outbreaks have been recorded in aquatic animals (Beleneva 2011), nor has L. mono- 
cytogenes infection ever been described in cetaceans, although $L$. ivanovii has been detected in pinnipeds (Thornton et al. 1998).

Toxoplasma gondii meningoencephalitis has been reported in odontocetes (Di Guardo et al. 2010), having been also detected in Brucella ceti-coinfected striped dolphins (González et al. 2002, Alba et al. 2013). Here we describe the first case of neurolisteriosis due to $L$. monocytogenes associated with $T$. gondii and Brucella spp. coinfection, in a cetacean species.

\section{METHODS AND RESULTS}

In January 2015, an adult female striped dolphin Stenella coeruleoalba was found dead on the Ligurian coast, northwestern Italy. The animal was $188 \mathrm{~cm}$ long, well-preserved (post mortem condition code 2; Geraci \& Lounsbury 2005) and emaciated. There was no evidence of interaction with fishing activities, and the stomach chambers were devoid of ingesta.
Furthermore, the dolphin showed a prominent subcutaneous oedema, along with blubber abscesses (Fig. 1A-C), multifocal splenic haemorrhage, severe Monorygma grimaldi infection and 2 Pholeter gastrophilus gastric nodules. All thoracic and abdominal lymph nodes were moderately enlarged and oedematous. Tissues were stored at both $4{ }^{\circ} \mathrm{C}$ and $-20^{\circ} \mathrm{C}$ for microbiological and biomolecular analyses and fixed in $10 \%$ buffered formalin for histopathology and immunohistochemistry (IHC).

Microscopically, nonsuppurative meningoencephalitis affecting the gray and white matter, mainly in the pons and thalamus, was observed. Vasculitis and perivascular cuffs characterized by lymphocytes and macrophages (Fig. 2A), along with gliosis, were the most represented lesions, associated with few microabscesses (Fig. 2B) and malacic areas. Choroiditis with few neutrophils was also apparent (Fig. 2C). In the cerebral cortex, scattered protozoan cysts surrounded by glial nodules and mononuclear cell aggregates were also observed. Presence of Toxoplasma gondii cysts was confirmed by IHC (Di
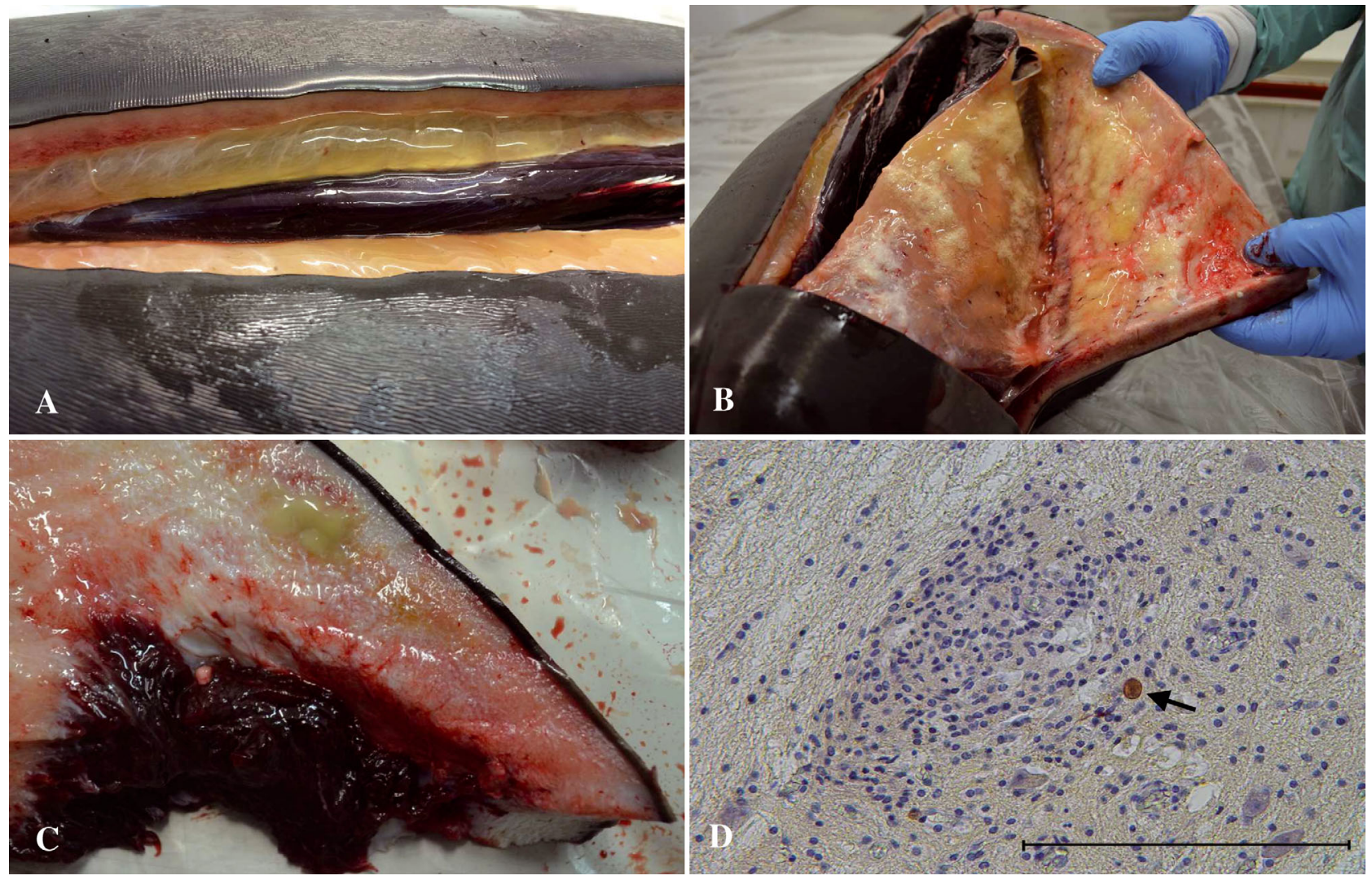

Fig. 1. Pathologic and immunohistochemical findings in a stranded adult female striped dolphin Stenella coeruleoalba. (A,B) Subcutaneous tissue with oedema. (C) Blubber with abscess. (D) Brain (thalamus), showing positive immunoreactivity for Toxoplasma gondii. A glial nodule close to a protozoan cyst is shown (arrow). Immunohistochemistry; scale bar $=100 \mu \mathrm{m}$ 


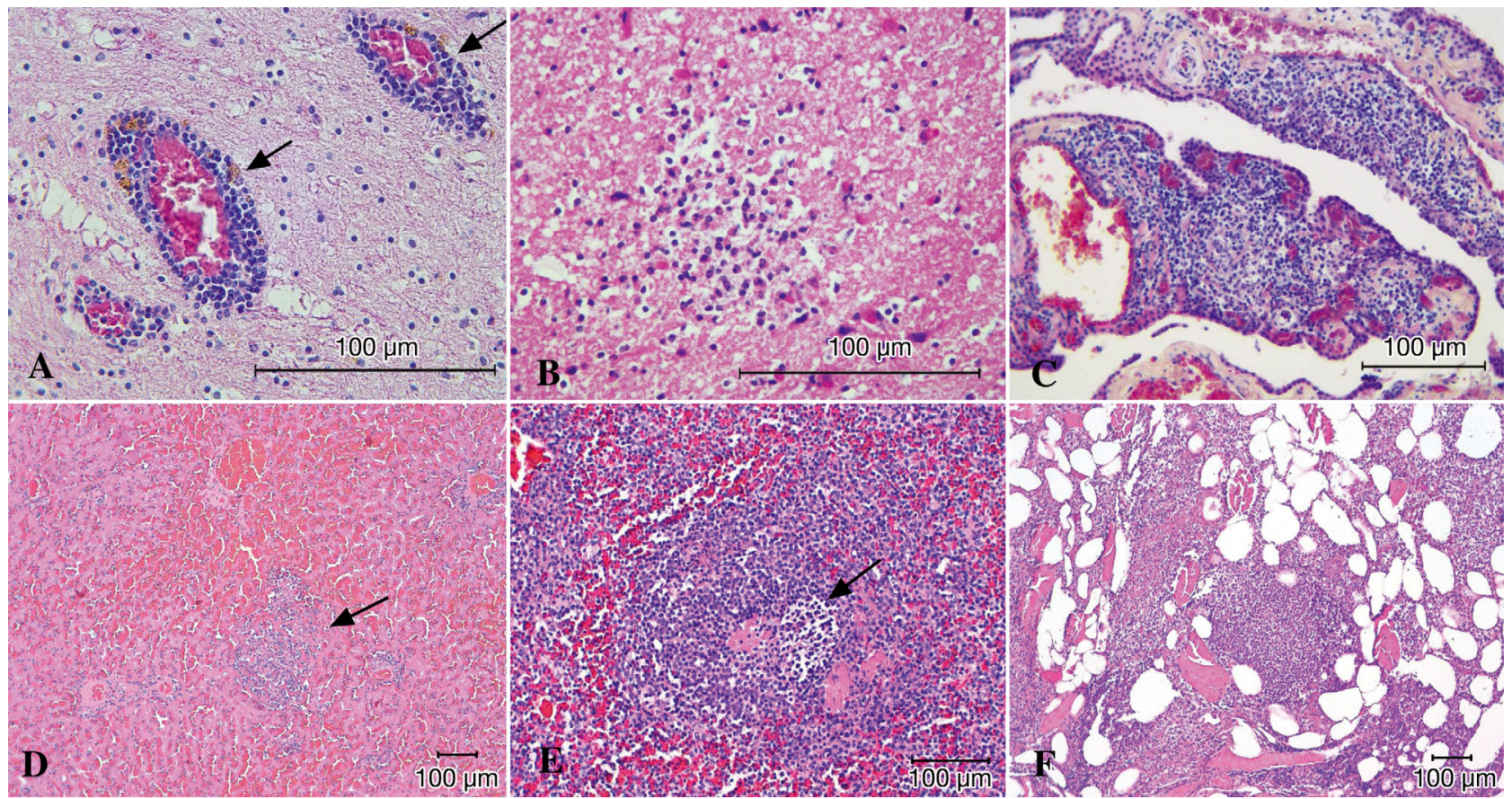

Fig. 2. Main histopathologic findings in a stranded adult female striped dolphin Stenella coeruleoalba. (A) Brain; thalamus. Perivascular cuffing with lymphocytes and macrophages containing haemosiderin-like pigment (arrows); haematoxylin-eosin (HE). (B) Brain; pons. Microabscesses with mononuclear cells and neutrophils; HE. (C) Brain; choroid plexus of fourth ventricle. Choroiditis characterized by mononuclear cells, rare neutrophils and focal collagen fibre thickening; HE. (D) Liver. Parenchymal necrosis, with infiltration of neutrophils and lymphocytes (arrow) and multifocal, chronic colangiohepatitis; HE. (E) Spleen. Follicular lymphocytic damage, along with intrafollicular hyalinosis (arrow) and hypercellularity (macrophages, plasma cells, neutrophils) in sinus areas; HE. (F) Blubber. Diffuse infiltration of neutrophils and macrophages; HE

Guardo et al. 2010; Fig. 1D), while IHC labelling for Listeria monocytogenes and morbillivirus, performed with polyclonal (Dell'Armelina Rocha et al. 2013) and monoclonal (Di Guardo et al. 2010) antibodies, respectively, did not demonstrate any antigen. The liver had multifocal necrosis and moderate chronic cholangiohepatitis (Fig. 2D). In the spleen (Fig. 2E) and multiple lymph nodes, we observed lymphocyte necrosis, follicular hyalinosis, sinusoidal histiocytosis with plasma cells and rare neutrophils and disperse karyrrhectic debris.

Pyogranulomatous inflammation was detected in blubber (Fig. 2F) and adrenal cortex. Furthermore, lung, kidney, skeletal muscle and intestine showed a mild, multifocal, chronic inflammation.

Polymerase chain reaction (PCR) (Mazzariol et al. 2012, Vitale et al. 2013) did not detect morbillivirus genomes and proved positive for T. gondii in brain, liver and prescapular lymph nodes. Evidence of Brucella spp. infection was obtained from brain, lung, spleen and blubber abscesses that were positive for the sequence bcsp31, using a hemi-nested PCR protocol to increase sensitivity and specificity (Baily et al. 1992, Bounaadja et al. 2009). Additional evidence of Brucella spp. infection was obtained by the National Reference Laboratory for Brucellosis from the lung that was positive to a TaqMan real-time PCR based on the multiple-copy Brucella-specific insertion sequence IS711. Each positive amplicon was confirmed for specificity through sequencing on an ABI PRISM 310 genetic analyzer.

Anti- T. gondii antibodies (1:320), with no simultaneous evidence of anti-morbillivirus antibodies, were detected in serum (Di Guardo et al. 2010). Furthermore, anti-Brucella spp. antibodies were detected by rapid serum agglutination, using $B$. abortus and B. melitensis as antigens (Hernández-Mora et al. 2008, Di Guardo et al. 2010).

Conventional bacteriological assays were performed under aerobic, anaerobic and microaerobic $\left(5 \% \mathrm{CO}_{2}\right)$ conditions on brain, lung, liver, spleen, mediastinal and prescapular lymph nodes, kidney and blubber abscesses. Specific bacteriological investigations following international recommendations (OIE 2015) were also carried out for Listeria spp. from the brain, as well as for Salmonella spp. from 
the liver and for Brucella spp. from the brain, spleen, lung, lymph nodes and blubber abscesses. Selective cultivation of Listeria spp. was performed by means of an enrichment step in liquid media, followed by subculture on solid media (Demi-Fraser broth, Microbiol; Fraser broth, Biolife; Listeria Aesculin Agar, Liofilchem) (Dell'Armelina Rocha et al. 2013, OIE 2015); the same approach was performed for Salmonella spp. isolation (Selenite Cystine broth, Microbiol; MKTTn broth, Liofilchem; BGA and XLD agar, Oxoid). For Brucella spp. detection, direct isolation was carried out on Farrell's and Modified Thayer Martin's solid media, while enrichment cultures were performed on Brucella enrichment broth (Oxoid), supplemented with foetal horse serum and modified Brucella selective supplement (Oxoid). Fungal cultures of the lung and brain on Saboraud agar (Microbiol) were also performed.

Aeromonas spp., Morganella morganii and Micrococcus spp. were isolated from brain, kidney and lung, respectively. No Salmonella spp., Brucella spp. (despite multiple attempts) or fungi were recovered. L. monocytogenes was isolated from the brain, then confirmed by $16 \mathrm{~S}$ rDNA sequencing (MicroSeq 500 system, Life Technologies) and further serotyped as serovar 1/2a (Denka Seiken). This isolate was subtyped as ST399, belonging to lineage II, by means of multi-locus sequence typing (MLST; Haase et al. 2014). A comparison with the MLST database (www. pasteur.fr/mlst) showed that strain ST399 had been previously identified in New Zealand, but no details were available about the source of the bacterium.

Considering the chemical stress related to organochlorine (OC) xenobiotics in Mediterranean cetaceans, PCBs, HCB and DDTs were measured in blubber, according to the Environmental Protection Agency method 8081/8082 with modifications (Marsili \& Focardi 1997), and toxicological stress was evaluated using a theoretical model (Marsili et al. 2004). The results, expressed in $\mathrm{ng}^{-1}$ lipid weight basis (PCBs: 784006.7; DDTs: 644495.4; HCB: 1112; canonical variable value $[\mathrm{CAN}]=6.04)$, confirmed the presence of hazardous levels of OC pollutants $(\mathrm{CAN}>0.47)$.

\section{DISCUSSION}

This is an interesting and unprecedented case of coinfection by 3 zoonotic pathogens, viz. Listeria monocytogenes, Brucella spp. and Toxoplasma gondii, in a striped dolphin, as well as the first case of neurolisteriosis in a cetacean species. That the brain tissue from the dolphin under study was the target of a mixed bacterial and protozoal infection is additionally supported by the cellular constituents and nature of the inflammatory infiltrates. Multifocal microabscessation and malacic areas in the brainstem are suggestive of L. monocytogenes infection, while mononuclear choroiditis is commonly found in neurobrucellosis, and meningitis, gliosis and perivascular cuffs are usually non-specific and may accompany various pathologies.

Although the transmission pathways of L. monocytogenes to the dolphin and in the marine environment remain unknown, lineage II is known to include human, environmental, farm and food isolates (Haase et al. 2014), so that different hypotheses can be drawn. The presence of Listeria spp. in marine waters is widely described in the literature (Boum'handi et al. 2007, Zaytseva et al. 2007, Beleneva 2011). The transmission of infection could likely derive from the consumption of infected or contaminated marine organisms (Bou-m'handi et al. 2007, Beleneva 2011); alternatively, the infection could originate from human and/or animal faecal contamination, potentially increased by the severe flooding that occurred on the Ligurian coast in October 2014, or from the release of contaminated ship waters. Concurrent Brucella spp. and T. gondii coinfection, along with the severe stress confirmed by the burdens of different persistent organic pollutants and by the emaciation, likely acted as predisposing factors for the development of the L. monocytogenes-associated brain lesions found in the dolphin under study. In this respect, while $B$. ceti frequently behaves as a primary neurotropic pathogen in striped dolphins (Hernández-Mora et al. 2008, Alba et al. 2013), the potential of mixed infections to give rise to more severe disease patterns in free-ranging cetaceans should also be emphasized. This is clearly exemplified by the frequent cases of dolphin morbillivirus (DMV)-T. gondii coinfection among Mediterranean striped dolphins affected by the dramatic DMV epidemic that occurred between 1990 and 1992 (Domingo et al. 1992), as well as by the cases of $T$. gondii-Sarcocystis neurona coinfection in wild marine mammals from the northeastern Pacific region, in which higher mortality rates and more severe encephalitic lesions were found in comparison to animals infected by T. gondii alone (Gibson et al. 2011).

This highlights the risk of neurolisteriosis for immunosuppressed cetaceans in the marine environment. On this basis, a systematic screening of stranded animals for $L$. monocytogenes would be necessary, in order to elucidate its pathogenicity, 
along with strain-specific virulence factors, clinical significance and transmission pathways of infection among cetaceans. Brucella spp. have not previously been detected along the Ligurian coast. The occurrence of related pathological changes in brain, blubber, liver and spleen (Tryland et al. 2005, HernándezMora et al. 2008, Nymo et al. 2011, Alba et al. 2013), coupled with the molecular identification of Brucella spp. in blubber, brain, spleen and lung and with seropositivity (albeit achieved using B. abortus and $B$. melitensis as antigens), suggest that this striped dolphin displayed a clinico-pathological disease phenotype. In this respect, our Brucella spp.-culturenegative but PCR-positive analyses could be related to a higher sensitivity of the PCR assay (Tryland et al. 2005), also strengthened by the detection of 2 DNA targets in the same sample, or to localized and/or peculiar forms of infection, which may hinder bacterial isolation (Tryland et al. 2005); moreover, given the detectable immune response, the animal could have developed a chronic infection phase (Sidor et al. 2013). T. gondii has recently become regarded as a primary neurotropic pathogen for striped dolphins (Di Guardo et al. 2010). The brain alterations observed around protozoan cysts and the extracerebral chronic lesions, along with the systemic molecular identification and $T$. gondii antibody-specific titres suggest a subacute to chronic $T$. gondii infection, a severe immune response impairment and an important pathogenic role in the protozoal encephalitis.

In conclusion, cerebral toxoplasmosis (Di Guardo et al. 2010, Mazzariol et al. 2012) and neurobrucellosis (Hernández-Mora et al. 2008, Nymo et al. 2011, Alba et al. 2013) may have led to the animal's disorientation and stranding, with L. monocytogenes supra-infection having likely exacerbated the disease condition, thus leading to the dolphin's death.

\section{LITERATURE CITED}

Alba P, Terracciano G, Franco A, Lorenzetti S and others (2013) The presence of Brucella ceti ST26 in a striped dolphin (Stenella coeruleoalba) with meningoencephalitis from the Mediterranean Sea. Vet Microbiol 164: 158-163

Baily GG, Krahn JB, Drasar BS, Stoker NG (1992) Detection of Brucella melitensis and Brucella abortus by DNA amplification. J Trop Med Hyg 95:271-275

Beleneva IA (2011) Incidence and characteristics of Staphylococcus aureus and Listeria monocytogenes from the Japan and South China seas. Mar Pollut Bull 62:382-387

Bou-m'handi N, Jacquet C, El Marrakchi A, Martin P (2007) Phenotypic and molecular characterization of Listeria monocytogenes strains isolated from a marine environment in Morocco. Foodborne Pathog Dis 4:409-417
Bounaadja L, Albert D, Chénais B, Hénault S, Zygmunt MS, Poliak S, Garin-Bastuji B (2009) Real-time PCR for identification of Brucella spp.: a comparative study of IS711, bcsp 31 and per target genes. Vet Microbiol 137:156-164

> Dell'Armelina Rocha PR, Lomonaco S, Bottero MT, Dalmasso A and others (2013) Ruminant rhombencephalitisassociated Listeria monocytogenes strains constitute a genetically homogeneous group related to human outbreak strains. Appl Environ Microbiol 79:3059-3066

$>$ Di Guardo G, Proietto U, Di Francesco CE, Marsilio F and others (2010) Cerebral toxoplasmosis in striped dolphins (Stenella coeruleoalba) stranded along the Ligurian sea coast of Italy. Vet Pathol 47:245-253

- Domingo M, Visa J, Pumarola M, Marco AJ, Ferrer L, Rabanal R, Kennedy S (1992) Pathologic and immunocytochemical studies of Morbillivirus infection in striped dolphins (Stenella coeruleoalba). Vet Pathol 29:1-10

Geraci JR, Lounsbury VJ (2005) Marine mammals ashore: a field guide for strandings, $2^{\text {nd }}$ edn. National Aquarium in Baltimore, Baltimore, MD

Gibson AK, Raverty S, Lambourn DM, Huggins J, Magargal SL, Grigg ME (2011) Polyparasitism is associated with increased disease severity in Toxoplasma gondiiinfected marine sentinel species. PLoS Negl Trop Dis 5: e1142

González L, Patterson IA, Reid RJ, Foster G and others (2002) Chronic meningoencephalitis associated with Brucella sp. infection in live-stranded striped dolphins (Stenella coeruleoalba). J Comp Pathol 126:147-152

Haase JK, Didelot X, Lecuit M, Korkeala H, L. monocytogenes MLST Study Group, Achtman M (2014) The ubiquitous nature of Listeria monocytogenes clones: a large-scale Multilocus Sequence Typing study. Environ Microbiol 16:405-416

Hernández-Mora G, Gonzáles-Barrientos R, Morales JA, Chaves-Olarte E and others (2008) Neurobrucellosis in stranded dolphins, Costa Rica. Emerg Infect Dis 14: 1430-1433

Marsili L, Focardi S (1997) Chlorinated hydrocarbons (HCB, DDTs and PCBs) levels in cetaceans stranded along the Italian coasts: an overview. Environ Monit Assess 45: 129-180

> Marsili L, D'Agostino A, Bucalossi D, Malatesta T, Fossi MC (2004) Theoretical models to evaluate hazard due to organochlorine compounds (OCs) in Mediterranean striped dolphin (Stenella coeruleoalba). Chemosphere 56:791-801

> Mazzariol S, Marcer F, Mignone W, Serracca L and others (2012) Dolphin Morbillivirus and Toxoplasma gondii coinfection in a Mediterranean fin whale (Balaenoptera physalus). BMC Vet Res 8:20

Nymo IH, Tryland M, Godfroid J (2011) A review of Brucella infection in marine mammals, with special emphasis on Brucella pinnipedialis in the hooded seal (Cystophora cristata). Vet Res 42:93

OIE (World Organisation for Animal Health) (2015) Manual of diagnostic tests and vaccines for terrestrial animals. OIE, Paris

Sidor IF, Dunn JL, Tsongalis GJ, Carlson J, Frasca S (2013) A multiplex real-time polymerase chain reaction assay with two internal controls for the detection of Brucella species in tissues, blood and feces from marine mammals. J Vet Diagn Invest 25:72-81

Thornton SM, Nolan S, Gulland FMD (1998) Bacterial isolates from California sea lions (Zalophus californianus), 
harbor seals (Phoca vitulina), and northern elephant seals (Mirounga angustirostris) admitted to a rehabilitation center along the central California coast, 1994-1995. J Zoo Wildl Med 29:171-176

Tryland M, Sørensen KK, Godfroid J (2005) Prevalence of Brucella pinnipediae in healthy hooded seals (Cystophora cristata) from the North Atlantic Ocean and ringed seals (Phoca hispida) from Svalbard. Vet Microbiol 105:103-111

Editorial responsibility: Michael Moore, Woods Hole, Massachusetts, USA
Vitale M, Galluzzo P, Currò V, Gozdzik K, Schillaci D, Di Marco Lo Presti V (2013) A high sensitive nested PCR for Toxoplasma gondii detection in animal and food samples. J Microb Biochem Technol 5:39-41

Zaytseva E, Ermolaeva S, Somov GP (2007) Low genetic diversity and epidemiological significance of Listeria monocytogenes isolated from wild animals in the far east of Russia. Infect Genet Evol 7:736-742

Submitted: July 8, 2015; Accepted: November 30, 2015

Proofs received from author(s): February 17, 2016 